\title{
A Review on Lignin Liquefaction: Advanced Characterization of Structure and Micro-kinetic Modeling
}

\author{
Evan Terrell ${ }^{1}$, Lauren D. Dellon ${ }^{2}$, Anthony Dufour ${ }^{3}$, Erika Bartolomei ${ }^{3}$, Linda J. Broadbelt ${ }^{2}$, \\ Manuel Garcia-Perez ${ }^{1 *}$ \\ ${ }^{1}$ Department of Biological Systems Engineering, Washington State University \\ Pullman, WA 99164, US \\ ${ }^{2}$ Department of Chemical and Biological Engineering, Northwestern University, Evanston, \\ Illinois, 60208, US \\ ${ }^{3}$ LRGP, CNRS, Universite de Lorraine, ENSIC, 54000 Nancy, France
}

Industrial and Engineering Chemistry Research

\section{Supporting Information}


Examples of Py-GC/MS monomer products originating from an $\mathrm{H}, \mathrm{S}$ or $\mathrm{G}$ monolignol

\begin{tabular}{|c|c|c|c|}
\hline $\begin{array}{c}\text { Compound } \\
\text { (H/S/G assignment) }\end{array}$ & Structure & $\begin{array}{c}\text { Compound } \\
\text { (H/S/G assignment) }\end{array}$ & Structure \\
\hline $\begin{array}{l}\text { Phenol } \\
\text { (H) }\end{array}$ & & $\begin{array}{l}\text { Homovanillic acid } \\
\text { (G) }\end{array}$ & \\
\hline $\begin{array}{l}\text { Cresols } \\
(\mathrm{H})\end{array}$ & & $\begin{array}{l}\text { Coniferyl alcohol } \\
\text { (G) }\end{array}$ & \\
\hline $\begin{array}{l}\text { Xylenols } \\
(\mathrm{H})\end{array}$ & & $\begin{array}{l}\text { Coniferaldehyde } \\
\text { (G) }\end{array}$ & \\
\hline $\begin{array}{l}\text { Propenylphenol } \\
\text { (H) }\end{array}$ & & $\begin{array}{l}\text { Syringol } \\
\text { (S) }\end{array}$ & \\
\hline $\begin{array}{l}\text { p-Coumaryl alcohol } \\
(\mathrm{H})\end{array}$ & & $\begin{array}{l}\text { Propylsyringol } \\
\text { (S) }\end{array}$ & \\
\hline $\begin{array}{l}\text { Guaiacol } \\
\text { (G) }\end{array}$ & & $\begin{array}{l}\text { Syringaldehyde } \\
\text { (S) }\end{array}$ & \\
\hline $\begin{array}{l}\text { Eugenol } \\
\text { (G) }\end{array}$ & & $\begin{array}{l}\text { Acetosyringone } \\
\text { (S) }\end{array}$ & \\
\hline $\begin{array}{l}\text { Vanillin } \\
\text { (G) }\end{array}$ & & $\begin{array}{l}\text { Sinapyl alcohol } \\
\text { (S) }\end{array}$ & \\
\hline
\end{tabular}



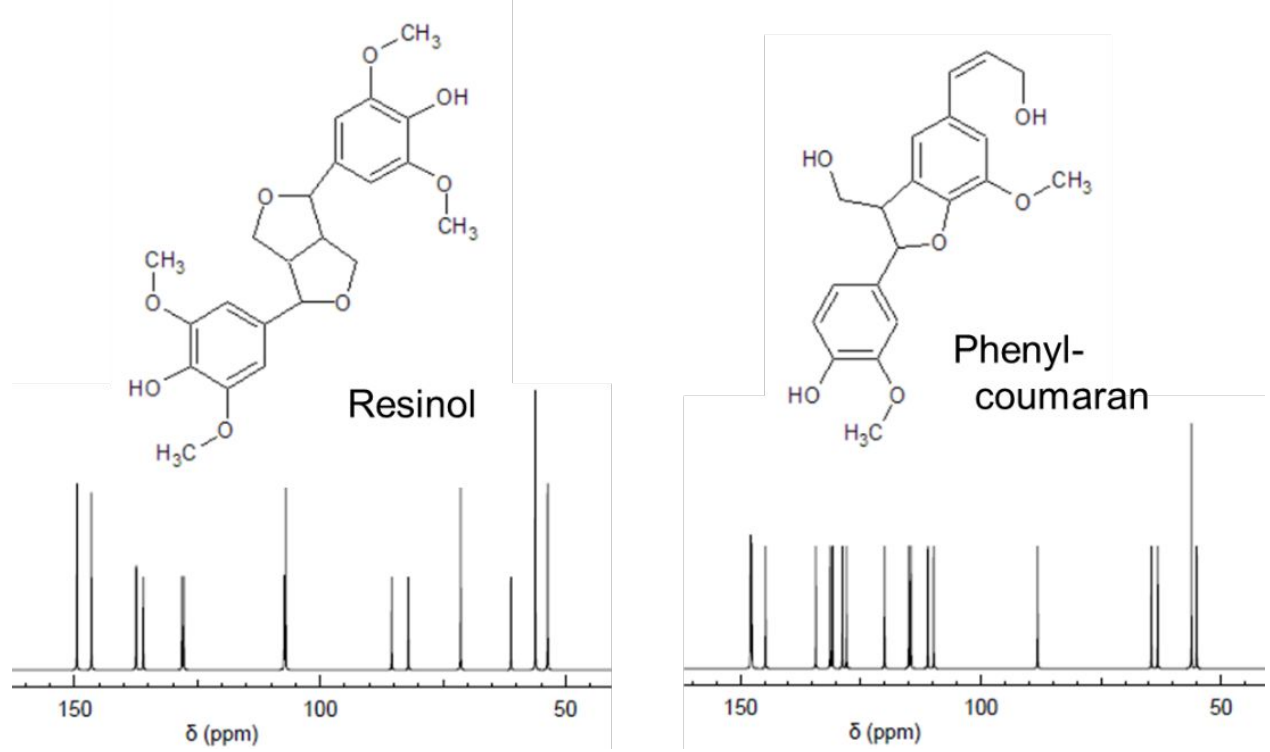

Illustration of an example in the differences between ${ }^{13} \mathrm{C}$ NMR spectra for different lignin subunits, resinol (left) and phenylcoumaran (right). Spectra were simulated using www.nmrdb.org. ${ }^{1 \text {, }}$ 2 
A selection of solvents and their corresponding Hildebrand parameters; adapted from Castellví Barnés, et al. and Buncel and Stairs. ${ }^{3,4}$

Solvent

(a) $\delta_{\mathrm{H}}=$ Hildebrand parameter, units $\mathrm{MPa}^{1 / 2}$; a value for lignin is given as $31.0 .{ }^{3}$ Note: The solubility parameter of a solvent mixture can be estimated by taking a weighted average of the parameters of the individual components in the mixture with respect to their volume fraction. 
References

(1) Castillo, A. M.; Patiny, L.; Wist, J. Fast and Accurate Algorithm for the Simulation of NMR Spectra of Large Spin Systems. J. Magn. Reson. 2011, 209 (2), 123-130. https://doi.org/10.1016/j.jmr.2010.12.008.

(2) Steinbeck, C.; Krause, S.; Kuhn, S. NMRShiftDB - Constructing a Free Chemical Information System with Open-Source Components. J. Chem. Inf. Comput. Sci. 2003, 43 (6), 1733-1739. https://doi.org/10.1021/ci0341363.

(3) Castellví Barnés, M.; Oltvoort, J.; Kersten, S. R. A.; Lange, J. P. Wood Liquefaction: Role of Solvent. Ind. Eng. Chem. Res. 2017, 56 (3), 635-644. https://doi.org/10.1021/acs.iecr.6b04086.

(4) Buncel, E.; Stairs, R. A. Solvent Effects in Chemistry, Second.; John Wiley \& Sons: Hoboken, New Jersey, 2016. 